$\underline{\text { Preprint typeset in JHEP style - HYPER VERSION }}$

DCPT-06/27

hep-th/0609224

\title{
A T-duality interpretation of the relationship between massive and massless magnonic TBA systems
}

\author{
Patrick Dorey \\ Department of Mathematical Sciences \\ University of Durham \\ Durham DH1 3LE, UK. \\ e-mail: p.e.dorey@durham.ac.uk \\ J. Luis Miramontes \\ Departamento de Física de Partículas, and \\ Instituto Gallego de Física de Altas Energías (IGFAE), \\ Universidad de Santiago de Compostela \\ 15782 Santiago de Compostela, Spain. \\ e-mail: miramont@usc.es
}

ABSTRACT: We propose an alternative understanding of the relationship between massive and massless magnonic TBA systems, using the T-duality symmetries of the Homogeneous sine-Gordon models. This is shown to be in agreement with a previous treatment by Dorey, Dunning and Tateo, based on the properties of Y-systems.

Keywords: Integrable Field Theories, Target-space duality, Thermodynamic Bethe Ansatz, Field Theories in Lower Dimensions, Non-linear Sigma Models. 


\section{Introduction.}

The Thermodynamic Bethe ansatz (TBA) [1] is one of the most effective ways to study the renormalization group $(\mathrm{RG})$ trajectories of two-dimensional integrable quantum field theories. It enables the exact ground state energy on a circle of circumference $R$ to be calculated from the solution of a system of coupled nonlinear integral equations, making it possible to study the theory at all length scales, by varying the value of $R$. The TBA equations can be deduced directly from the factorised $S$-matrix of the theory, but this procedure becomes complicated when the scattering is non-diagonal. This has often motivated an alternative tactic, starting by the construction of a sensible set of TBA equations, and investigating afterwards whether it corresponds to some two-dimensional theory, typically defined by an action of the form [2]

$$
S=S_{C F T}+\mu \int d^{2} x \Phi(x) .
$$

Here, $S_{C F T}$ denotes an action for a conformal field theory (CFT) that governs the ultraviolet (UV) behaviour, $\mu$ is a dimensionful coupling, and $\Phi$ is a perturbing operator.

Following this approach, a class of TBA systems whose structure is encoded in a product of two simply-laced Dynkin diagrams was constructed in [3] (see also [4, 5]), and they were conjectured to describe a variety of integrable perturbed coset CFTs. They include, as particular cases, many TBA systems previously considered by other authors: in particular, those describing the well-studied perturbations of the unitary minimal models $\mathcal{M}_{p}$ for $p=3,4, \ldots$ by their least relevant primary fields $\Phi_{1,3}$ [6, []. In the construction of [3], there is a particle and a TBA equation for each node of the product diagram. However, when the TBA system corresponds to a nondiagonal $S$-matrix, some of these particles are fictitious and carry no energy and no momentum. They are called magnons, and it is common to refer to such TBA systems as magnonic. Magnonic TBA systems often admit massive and massless versions. In both cases, they give rise to RG trajectories starting in the UV fixed point specified by $S_{C F T}$. Then, either the trajectory flows to some massive theory or it comes to another fixed point in the infrared, depending on whether the system is massive or massless, respectively. In many cases the massive and massless versions of a given magnonic TBA system correspond to the same action (1.1) for different signs of the coupling constant. This was originally noticed in [7], where the TBA system for $\mathcal{M}_{p}+\mu \Phi_{1,3}$ with $\mu>0$ was constructed. It is associated to $A_{p-2} \times A_{1}$, and it turned out to be massless, in contrast to the system that describes the regime with $\mu<0$ which is massive [6]. However, there are also cases where the massive and massless TBA systems are not related by continuation in $\mu$. Examples are provided by the models $H_{N}^{(0)}$ (massive) and $H_{N}^{(\pi)}$ (massless) of $[8]$, which are related 
by analytic continuation for $N$ odd, but not for $N$ even. In the classification of [3], they are associated to $D_{N} \times A_{1}$.

This issue was clarified in [9], where it was pointed out the transformation could be understood directly in terms of the analytic continuation of the corresponding TBA systems under $\mu \rightarrow-\mu$. The massive and massless TBA systems corresponding to the same product diagram are known to be related in a very simple way that relies on the existence of a $\mathbb{Z}_{2}$ symmetry of the associated Dynkin diagrams. The authors of [9] pointed out that the continuation $\mu \rightarrow-\mu$ will change a massive TBA system into a massless one provided that the $\mathbb{Z}_{2}$ symmetry used in its construction coincides with the $\mathbb{Z}_{2}$ symmetry that characterises the periodicity properties of the associated Y-system. This systemetised the previously-known zoology of examples in a simple rule, and also provided a conceptual understanding, from the TBA point of view, of why such a rule should exist.

The purpose of this letter is to propose an alternative understanding of the relationship between the continuation $\mu \rightarrow-\mu$ and the transformation between massive and massless TBA systems that does not rely on the properties of the Ysystems. It will be deduced in the context of the Homogeneous sine-Gordon (HSG) theories [11, 12, 13] by making use of Lagrangian methods. The TBA equations of the HSG theories [14, 15] are purely massive generalisations of the magnonic TBA systems corresponding to products of the form $G \times A_{k-1}$. Moreover, they admit a Lagrangian formulation in terms of perturbed gauged Wess-Zumino-Witten (WZW) models where the required relationship arises as a consequence of their target-space duality (T-duality) symmetries [16].

The letter is organised as follows. In section 2, we review the main features of the TBA systems constructed in [3] and their associated Y-systems. For completeness, section 3 explains the TBA argument of [9, 10], relating the continuation $\mu \rightarrow-\mu$ to the transformation between massive and massless magnonic systems. In section 1 , we elucidate the relationship between the TBA systems of the HSG theories and the TBA systems constructed in [3]. The HSG TBA equations depend on a set of independent adjustable parameters, and their $\mu \rightarrow-\mu$ continuation is shown to be equivalent to a transformation among those parameters. Then, in section 5, we make use of the Lagrangian formulation of the HSG theories to show that the same equivalence arises as a consequence of T-duality. Moreover, this enables the resulting transformation among the parameters to be written in terms of a particular element of the Weyl group of $G$. In the context of the HSG theories, the magnonic massive and massless systems of [3] arise as the effective TBA systems describing particular crossovers [14. Thus, this correspondence points out a novel interpretation of the relationship between the continuation $\mu \rightarrow-\mu$ and the transformation between massive and massless TBA systems as a manifestation of T-duality, which constitutes our main result. Finally, section 6 contains our conclusions. 


\section{Magnonic TBA equations and Y-systems.}

The structure of the magnonic TBA systems constructed in [3] is encoded in the product $G \times H$ of two simply-laced Dynkin diagrams $G$ and $H$. We will denote by $r_{G}$ and $r_{H}$ the ranks of the corresponding algebras, and by $h_{G}$ and $h_{H}$ their Coxeter numbers. For each node of the resulting product diagram, there is a pseudoenergy $\varepsilon_{a}^{i}(\theta)$ and energy term $\nu_{a}^{i}(\theta)$. Defining $L_{a}^{i}(\theta)=\ln \left(1+\mathrm{e}^{-\varepsilon_{a}^{i}(\theta)}\right)$, the system of TBA equations is

$$
\nu_{a}^{i}(\theta)=\varepsilon_{a}^{i}(\theta)+\sum_{b=1}^{r_{H}}\left(\phi_{a b} * L_{b}^{i}(\theta)-\sum_{j=1}^{r_{G}} G_{i j} \psi_{a b} * L_{b}^{j}(\theta)\right),
$$

for $i=1 \ldots r_{G}$ and $a=1 \ldots r_{H}$. In these equations, ' $*$ ' denotes the usual rapidity convolution $f * g(\theta)=\int_{-\infty}^{+\infty} \frac{d \theta^{\prime}}{2 \pi} f\left(\theta-\theta^{\prime}\right) g\left(\theta^{\prime}\right)$, and $G_{i j}$ is the incidence matrix of $G$. Similarly, we will call $H_{a b}$ the incidence matrix of $H$. The TBA kernels can be written as

$$
\phi_{a b}=-i \frac{d}{d \theta} \ln S_{a b}^{\mathrm{min}}, \quad \psi_{a b}=-i \frac{d}{d \theta} \ln S_{a b}^{\mathrm{F}}
$$

in terms of the functions

$$
S_{a b}^{\min }=\prod_{x \in A_{a b}}\{x\}, \quad S_{a b}^{\mathrm{F}}=\prod_{x \in A_{a b}}(x),
$$

where $S_{a b}^{\min }$ are the minimal parts of the affine Toda $S$-matrix elements corresponding to $H$. Here, $A_{a b}$ is a set of integer numbers (possibly with repetitions), ${ }^{1}$ and the basic blocks read

$$
\{x\}=(x-1)(x+1), \quad(x) \equiv(x)(\theta)=\frac{\sinh \frac{1}{2}\left(\theta+i \pi x / h_{H}\right)}{\sinh \frac{1}{2}\left(\theta-i \pi x / h_{H}\right)}
$$

More details about the definitions of $S_{a b}^{\mathrm{min}}$ and $S_{a b}^{\mathrm{F}}$ can be found in 17 .

The TBA expression for the ground state energy of the system on a circle of circumference $R$ is then

$$
E_{0}(R)=E_{\text {bulk }}(M, R)-\pi c(r) / 6 R,
$$

where $c(r)$ is the so-called effective central charge, which can be calculated in terms of the solutions to the TBA equations,

$$
c(r)=\frac{3}{\pi^{2}} \sum_{i=1}^{r_{G}} \sum_{a=1}^{r_{H}} \int_{-\infty}^{+\infty} d \theta \nu_{a}^{i}(\theta) L_{a}^{i}(\theta)
$$

and $E_{b u l k}(M, R)$ is a bulk term. Here, $M$ is a mass scale, $r=M R$ a dimensionless overall scale, and the dependence on $R$ and on any other mass scale in the theory enters via the energy terms $\nu_{a}^{i}(\theta)$.

\footnotetext{
${ }^{1}$ For $H=A_{k}$, this set is $A_{a b}=\{a+b+1-2 l \mid l=1 \ldots \min (a, b)\}=\{|a-b|+1 \ldots a+b-1$, step 2$\}$.
} 
For each choice of $G \times H$, the authors of [3] defined $r_{G}$ different massive magnonic TBA systems, one for each node $l=1 \ldots r_{G}$ of the Dynkin diagram $G$, by choosing energy terms of the form

$$
\nu_{a}^{i}(\theta)=\delta_{i, l} \mu_{a} r \cosh \theta,
$$

where $\mu_{a}$ are the components of the Perron-Frobenius eigenvector of the Cartan matrix of $H$. The form of $\nu_{a}^{i}(\theta)$ reflects the particle spectrum of the theory, which in this case consists of $r_{H}$ massive particles attached to the nodes $(l, a)$ for $a=1 \ldots r_{H}$. Their masses are given by $M_{a}^{(l)}=M \mu_{a}$, with $M$ an overall mass scale. The particles that could be associated to all the other nodes of $G \times H$ are magnons, and they only contribute indirectly to $c(r)$ and $E_{0}(R)$, via their effects on the non-magnonic pseudoenergies $\varepsilon_{a}^{l}(\theta)$.

In contrast, as anticipated in the introduction, the massless magnonic systems of [3] require the existence of a $\mathbb{Z}_{2}$ symmetry. Let us suppose that the Dynkin diagram $G$ has a $\mathbb{Z}_{2}$ symmetry $\omega: G \rightarrow G$ that relates two nodes $l$ and $l^{\prime}=\omega(l) \neq l$. Then, we can associate a massless magnonic TBA system to the node $l$ by choosing the energy terms as follows:

$$
\nu_{a}^{i}(\theta)=\delta_{i, l} \frac{1}{2} \mu_{a} r \mathrm{e}^{-\theta}+\delta_{i, \omega(l)} \frac{1}{2} \mu_{a} r \mathrm{e}^{+\theta} .
$$

In this case, the particle spectrum of the theory consists of $2 r_{H}$ massless particles: $r_{H}$ left-movers and $r_{H}$ right-movers associated with the nodes $(l, a)$ and $(\omega(l), a)$, respectively. This exhibits that $M$ is a crossover scale in this case. Again, the particles associated to all the other nodes in $G \times H$ are magnons. All the massless TBA systems that have been discovered to date are related to massive systems by means of a transformation of the energy terms similar to the one that takes (2.7) into (2.8) [3, 9], a process which can sometimes be quite elaborate [18].

An important feature of the TBA equations is that they provide ( $r$-dependent) solutions to a set of functional algebraic equations called the Y-system 19. The Y-system corresponding to (2.1) is

$$
Y_{a}^{i}\left(\theta+\frac{i \pi}{h_{H}}\right) Y_{a}^{i}\left(\theta-\frac{i \pi}{h_{H}}\right)=\prod_{b=1}^{r_{H}}\left(1+Y_{b}^{i}(\theta)\right)^{H_{a b}} \prod_{j=1}^{r_{G}}\left(1+\frac{1}{Y_{a}^{j}(\theta)}\right)^{-G_{i j}},
$$

with $Y_{a}^{i}(\theta)=\mathrm{e}^{\varepsilon_{a}^{i}(\theta)}$ an entire function of $\theta$. Notice that the Y-system is completely independent of the form of the energy terms and, in particular, of the value of the dimensionless scale $r$. The role of the energy terms is to fix the asymptotic behaviour of $Y_{a}^{i}(\theta)$. Indeed, since $Y_{a}^{i}=\mathrm{e}^{\varepsilon_{a}^{i}}$, it is controlled by the asymptotic behaviour of $\epsilon_{a}^{i}(\theta)$ that, in turn, is dominated by the energy term $\nu_{a}^{i}(\theta)$. In particular, the asymptotic behaviour of the solutions to the massive TBA system specified by (2.7) is

$$
Y_{a}^{i}(\theta) \stackrel{\theta \rightarrow \pm \infty}{\longrightarrow} \begin{cases}\exp \left(\frac{1}{2} \mu_{a} r \mathrm{e}^{ \pm \theta}\right) \approx \exp \left(\nu_{a}^{l}(\theta)\right) & \text { for } i=l, \\ y_{a}^{i} & \text { for } i \neq l,\end{cases}
$$


where $y_{a}^{i}$ are the solutions to the constant ( $\theta$-independent) Y-system

$$
\left(y_{a}^{i}\right)^{2}=\prod_{b=1}^{r_{H}}\left(1+y_{b}^{i}\right)^{H_{a b}} \prod_{j=1}^{r_{G}}\left(1+\frac{1}{y_{a}^{j}}\right)^{-G_{i j}}
$$

In contrast, the asymptotic behaviour of the solutions to the massless TBA system whose energy terms are (2.8) is given by

$$
\begin{gathered}
Y_{a}^{i}(\theta) \stackrel{\theta \rightarrow-\infty}{\longrightarrow} \begin{cases}\exp \left(\frac{1}{2} \mu_{a} r \mathrm{e}^{-\theta}\right) & \text { for } \quad i=l, \\
y_{a}^{i} & \text { for } i \neq l,\end{cases} \\
\stackrel{\theta \rightarrow+\infty}{\longrightarrow} \begin{cases}\exp \left(\frac{1}{2} \mu_{a} r \mathrm{e}^{+\theta}\right) & \text { for } i=\omega(l), \\
y_{a}^{i} & \text { for } i \neq \omega(l) .\end{cases}
\end{gathered}
$$

It is important to stress that fixing the asymptotic behaviour of the Y-functions is not quite enough to ensure that the solutions to the Y-system (2.9) correspond to solutions to the ground-state TBA equations (2.1). The reason is that a given Y-system admits more solutions than those related to the original TBA equations. In fact, the same Y-system describes different excited states of the model, and the difference between the various excited state solutions is in their analytical structure [20, 21, 22, 23]. The simplest case concerns the ground state itself, which provides the solution to the original TBA system. It is recovered by restricting ourselves to Y-functions which are free of zeroes in the strip $-\pi / h_{H}<\operatorname{Im}(\theta)<\pi / h_{H}$. With this restriction, the Y-system (2.9) with appropriate asymptotic behaviour is completely equivalent to the system of TBA equations (2.1).

One of the main properties of Y-systems, first noticed in [19, is that they generate periodic functions. In our case the Y-functions satisfy [3, 24]

$$
Y_{a}^{i}(\theta+i \pi P)=Y_{\bar{a}}^{\bar{\imath}}(\theta) \quad \text { with } \quad P=\frac{h_{G}+h_{H}}{h_{H}}
$$

where the nodes $\bar{\imath}$ and $\bar{a}$ are conjugate to the nodes $i$ and $a$ on the Dynkin diagrams $H$ and $G$, respectively, and conjugation acts on Dynkin diagrams in the same way as charge conjugation acts on the particles in an affine Toda field theory (see fig. 1). The period $P$ can then be related to the conformal dimension of the perturbing operator $\Phi$; see [19, 3] for more details.

Eq. (2.13) was originally verified by direct successive substitutions in (2.9) for particular (low rank) choices of $G \times H$. Subsequently, the periodicity for the cases of the form $G \times A_{1}$ was proved in 25] $\left(G=A_{n}\right)$, [26] $\left(G=D_{n}\right)$, and 27]. Proofs for $A_{m} \times A_{n}$ with $m, n \neq 1$ have been recently provided in [28]. 


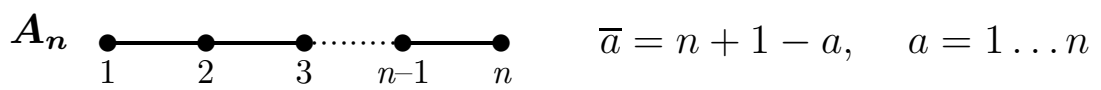

$$
\begin{aligned}
& \begin{array}{l}
\boldsymbol{D}_{\boldsymbol{n}} \\
\hdashline
\end{array}
\end{aligned}
$$

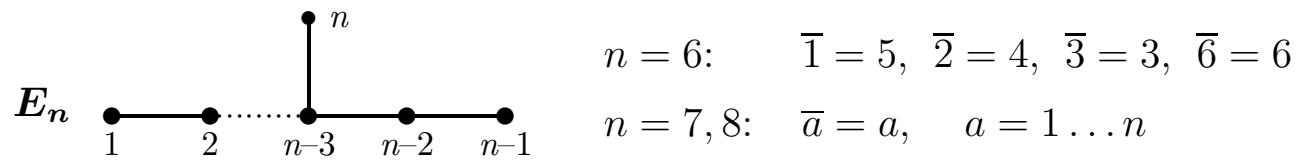

Figure 1: Dynkin diagrams of the simply-laced Lie algebras. The numbers show our labelling convention for the nodes. The explicit form of the conjugation that appear in eq. (2.13) has also been included.

\section{The $\mu \rightarrow-\mu$ continuation of the TBA equations.}

We are now in a position to give the TBA argument of [9, 10], relating the changes from massive into massless magnonic TBA systems to the continuation $\mu \rightarrow-\mu$ of the corresponding actions. Consider the massive magnonic TBA system specified by (2.7), and assume that it corresponds to some two-dimensional action of the form (1.1). On dimensional grounds, the coupling constant $\mu$ is related to the mass scale $M$ as

$$
\mu=\kappa M^{\frac{2}{P}}
$$

with $\kappa$ a dimensionless (non-perturbative) constant. Correspondingly, the dimensionless function $F_{0}(r)=R E_{0}(R) / 2 \pi$ is expected to be a regular function of $r^{\frac{2}{P}}$, which suggests that the TBA system of the same theory with $\mu \rightarrow-\mu$ can be obtained by putting $r=M R$ on the ray

$$
r=\mathrm{e}^{i \frac{\pi P}{2}} \rho, \quad \rho \in \mathbb{R}^{+},
$$

where $\rho$ is the dimensionless overall scale of the resulting theory. Notice that this transformation makes the energy terms (2.7) complex. However, the explicit calculations presented in [22] for the scaling Lee-Yang model support the expectation that the ground-state scaling function $F_{0}(r)$ evaluated at $r=\mathrm{e}^{i \frac{\pi P}{2}} \rho$ is real up to some value $\rho_{0}$ where it exhibits a branch point. And, moreover, that its value indeed corresponds to the ground state of the theory with $\mu \rightarrow-\mu$ for $\rho=|r|<\rho_{0}$. In the following, we will assume that a similar result holds in general.

The idea of [9, 10] is to consider the massive magnonic TBA system specified by (2.7), and to study the effect of (3.2) on the corresponding Y-system, in the

spirit of [22, 23]. The analytically continued Y-functions will also be solutions to the 
original Y-system, but with a different asymptotic behaviour. In the following, it will be useful to display the dependence of the solutions to the Y-system on $r$ : $Y_{a}^{i}(\theta) \equiv$ $Y_{a}^{i}(r, \theta)$. Then, the form of the transformed energy terms implies that the analytically continued Y-functions have the following real-valued asymptotic behaviour

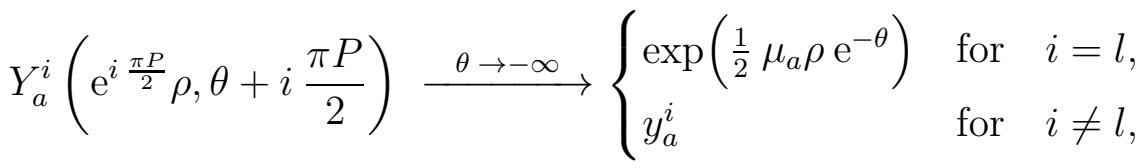

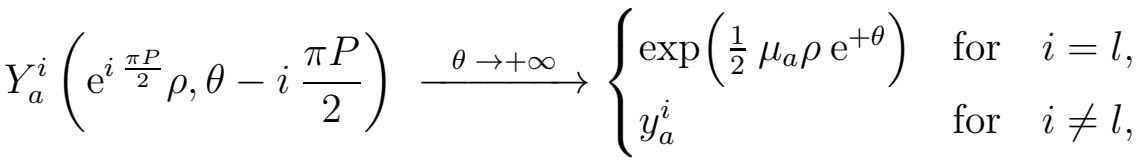

and, taking the periodicity (2.13) into account,

$$
\begin{aligned}
Y_{a}^{i}\left(\mathrm{e}^{i \frac{\pi P}{2}} \rho, \theta+i \frac{\pi P}{2}\right)= & Y_{\bar{a}}^{\bar{\imath}}\left(\mathrm{e}^{i \frac{\pi P}{2}} \rho, \theta-i \frac{\pi P}{2}\right) \\
& \stackrel{\theta \rightarrow+\infty}{\longrightarrow} \begin{cases}\exp \left(\frac{1}{2} \mu_{a} \rho \mathrm{e}^{+\theta}\right) & \text { for } i=\bar{l}, \\
y_{a}^{i} & \text { for } i \neq \bar{l},\end{cases}
\end{aligned}
$$

where we have used that $\overline{\bar{l}}=l$, and that $\mu_{\bar{a}}=\mu_{a}$.

If we compare (3.3) and (3.5) with (2.12), we observe that

$$
\widetilde{Y}_{a}^{i}(\rho, \theta)=Y_{a}^{i}\left(\mathrm{e}^{i \frac{\pi P}{2}} \rho, \theta+i \frac{\pi P}{2}\right) \equiv \mathrm{e}^{\widetilde{\varepsilon}_{a}^{i}(\rho, \theta)}
$$

provides a solution to the massless magnonic TBA system specified by the energy terms

$$
\nu_{a}^{i}(\theta)=\delta_{i, l} \frac{1}{2} \mu_{a} \rho \mathrm{e}^{-\theta}+\delta_{i, l} \frac{1}{2} \mu_{a} \rho \mathrm{e}^{+\theta} .
$$

In other words, the massive magnonic TBA system defined by (2.7) is related to the massless system specified by (2.8) by means of the continuation $\mu \rightarrow-\mu$ provided that the $\mathbb{Z}_{2}$ symmetry used in the construction of the latter coincides with the conjugation that characterises the periodicity conditions of the Y-functions; i.e., $\omega(l)=\bar{l} .^{2}$

${ }^{2}$ The same arguments applied to the analytical continuation $r \rightarrow \mathrm{e}^{i \pi P} r$ lead to

$$
Y_{a}^{i}\left(\mathrm{e}^{i \pi P} r, \theta+i \pi P\right)=Y_{a}^{i}(r, \theta),
$$

which is an identity originally obtained in [23, eq. 2.8]. There, it was deduced as a consequence of the claim that $Y_{a}^{i}(r, \theta)$ can be expanded as a power series in the two variables $a_{ \pm}=\left(r \mathrm{e}^{ \pm \theta}\right)^{\frac{1}{P}}$ with a finite domain of convergence about $a_{+}=a_{-}=0$. Taking (3.1) into account, this analytic continuation corresponds to leaving $\mu$ invariant. 


\section{4. $\mu \rightarrow-\mu$ continuation in the HSG models.}

An alternative understanding of the relation between the continuation $\mu \rightarrow-\mu$ of the perturbed CFT action and the transformation changing a massive magnonic TBA system into a massless one can be obtained by considering the Lagrangian formulation of the simply-laced Homogeneous sine-Gordon (HSG) theories. This will be discussed in the next section. But first, we clarify the relationship between the HSG TBA equations and the magnonic TBA systems of [3].

The Homogeneous sine-Gordon theories are integrable perturbations of level $k$ $G$-parafermions, that is of coset CFTs of the form $G_{k} / U(1)^{r_{G}}$, where $G$ is a simple compact Lie group, $k>1$ is an integer, and $r_{G}$ is the rank of $G$ [12]. In the following, we will use $G$ to denote both the Lie group and the Dynkin diagram of its Lie algebra, and we will restrict ourselves to the case of simply-laced $G$. The exact $S$-matrices of the simply-laced HSG theories have been constructed in [13] (see also [14, 15]). They are always diagonal and describe the scattering of a set of stable solitonic massive particles labelled by two quantum numbers, $(i, a)$, where $i=1 \ldots r_{G}$ and $a=1 \ldots k-1$. In other words, there is a stable particle for each node of $G \times A_{k-1}$, the product of the Dynkin diagrams $G$ and $A_{k-1}$. The mass of the particle $(i, a)$ is

$$
M_{a}^{i}=M m_{i} \mu_{a}
$$

where $M$ is a dimensionful overall mass scale, $m_{1} \ldots m_{r_{G}}$ are $r_{G}$ arbitrary nonvanishing relative masses, one for each node of the Dynkin diagram $G$, and $\mu_{a}=$ $\sin (\pi a / k)$ are the components of the Perron-Frobenius eigenvector of the $A_{k-1}$ Cartan matrix. The $S$-matrix elements depend on a further set of real resonance parameters $\sigma_{i j}=-\sigma_{j i}$ defined for each pair $\{i, j\}$ of neighbouring nodes on $G$. They are most conveniently specified by assigning a variable $\sigma_{i}$ to each node of $G$ and setting $\sigma_{i j}=\sigma_{i}-\sigma_{j}$. The resulting set of parameters $M,\left\{m_{i}\right\}$, and $\left\{\sigma_{i}\right\}$ is redundant, but the obvious symmetries $M \rightarrow \alpha M,\left\{m_{i} \rightarrow \alpha^{-1} m_{i}\right\}$, and $\left\{\sigma_{i} \rightarrow \sigma_{i}+\beta\right\}$ ensure that there are $2 r_{G}-1$ independent adjustable parameters, which is one of the interesting features of these theories.

The TBA equations of the HSG models have the standard form for a diagonal scattering theory, although care is needed in their derivation because parity is not a symmetry [15]. There is a pseudoenergy $\widehat{\epsilon}_{a}^{i}(\theta)$ for each of the $(k-1) \times r_{G}$ stable particles, and the mass scales influence them via $(k-1) \times r_{G}$ energy terms

$$
\widehat{\nu}_{a}^{i}(\theta)=M_{a}^{i} R \cosh \theta=m_{i} \mu_{a} r \cosh \theta
$$

where $r=M R$. Using the same conventions of (2.1), the pseudoenergies solve the TBA equations

$$
\widehat{\nu}_{a}^{i}(\theta)=\widehat{\epsilon}_{a}^{i}(\theta)+\sum_{b=1}^{k-1}\left(\phi_{a b} * \widehat{L}_{b}^{i}(\theta)-\sum_{j=1}^{r_{G}} G_{i j} \psi_{a b} * \widehat{L}_{b}^{j}\left(\theta-\sigma_{j}+\sigma_{i}\right)\right) .
$$


Then, the dimensionless effective central charge $c(r)$ is expressed in the usual way by (2.6), with $r_{H}=k-1$. Its limiting value as $r \rightarrow 0$ with all the other parameters fixed was calculated in [15], with the result

$$
\lim _{r \rightarrow 0} c(r)=\frac{k-1}{k+r_{G}} h_{G} r_{G},
$$

which is the central charge of the $G_{k} / U(1)^{r_{G}}$ coset CFT. This holds for any fixed choice of the mass scales $0<m_{i}<+\infty$ and resonance parameters $-\infty<\sigma_{i}<$ $+\infty$. Other exact multiple scaling limits, where the parameters $m_{i}$ and $\sigma_{i}$ approach particular limiting values while $r \rightarrow 0$, have been discussed in [14]. In the opposite, $r \rightarrow+\infty$, limit, $c(r)$ tends to zero, as expected for a massive theory.

In order to emphasise the similarities of the HSG TBA systems with the magnonic TBA systems of [3], it is convenient to eliminate the explicit dependence of the TBA equations on the resonance parameters by writing them in terms of

$$
\varepsilon_{a}^{i}(\theta)=\widehat{\varepsilon}_{a}^{i}\left(\theta-\sigma_{i}\right) .
$$

Then, (4.3) becomes

$$
\nu_{a}^{i}(\theta)=\varepsilon_{a}^{i}(\theta)+\sum_{b=1}^{k-1}\left(\phi_{a b} * L_{b}^{i}(\theta)-\sum_{j=1}^{r_{G}} G_{i j} \psi_{a b} * L_{b}^{j}(\theta)\right),
$$

where

$$
\nu_{a}^{i}(\theta)=\widehat{\nu}_{a}^{i}\left(\theta-\sigma_{i}\right)=\frac{1}{2} m_{i}^{+} \mu_{a} r \mathrm{e}^{-\theta}+\frac{1}{2} m_{i}^{-} \mu_{a} r \mathrm{e}^{+\theta},
$$

and we have introduced

$$
m_{i}^{ \pm}=m_{i} \mathrm{e}^{ \pm \sigma_{i}} .
$$

Eq. (4.6) is identical to (2.1) for $H=A_{k-1}$, which exhibits that the HSG TBA systems corresponding to perturbations of the $G / U(1)^{r_{G}}$ coset CFT are massive versions of the TBA systems constructed in [3] in terms of the product $G \times A_{k-1}$. The explicit asymmetric $\theta \rightarrow-\theta$ structure of the energy terms (4.7) indicates that the HSG theories are not parity symmetrical in general.

Formally, the magnonic $G \times A_{k-1}$ TBA systems of [3] could be recovered from (4.6) by suitably choosing the arbitrary parameters $m_{i}^{+}$and $m_{i}^{-}$. Namely, $m_{i}^{ \pm}=\delta_{i, l}$ for the massive system specified by (2.7), and $m_{i}^{-}=\delta_{i, l}$ together with $m_{i}^{+}=\delta_{i, \omega(l)}$ for the massless system whose energy terms are (2.8). However, since the HSG theories are purely massive these choices are not permitted. In general, the connection between the massive HSG, and the magnonic $G \times A_{k-1}$ TBA systems is recovered in a different, more subtle, way: the latter are the effective TBA systems describing the crossovers of the HSG theories for particular limiting values of their parameters. Crossover phenomena in the HSG theories were discussed in detail in [14]. One of the 
main results of that paper is that the HSG theories exhibit a crossover at $r \approx 2 / m_{p q}$ for each 'unshielded' mass scale $m_{p q}$ within the set of numbers given by

$$
m_{p q}=\sqrt{m_{p} m_{q}} \mathrm{e}^{\left(\sigma_{q}-\sigma_{p}\right) / 2}=\sqrt{m_{q}^{+} m_{p}^{-}}, \quad p, q=1 \ldots r_{G} .
$$

Moreover, when the unshielded scale $m_{p q}$ is 'well separated' from the others, the crossover at $r \approx 2 / m_{p q}$ is described by an effective TBA system of the form (4.6) with energy terms

$$
\nu_{a}^{i}(\theta)=\delta_{i, p} \frac{1}{2} m_{p q} \mu_{a} r \mathrm{e}^{-\theta}+\delta_{i, q} \frac{1}{2} m_{p q} \mu_{a} r \mathrm{e}^{+\theta}
$$

(see [14] for details). For $p=q \equiv l$, and $p=\omega(q) \equiv l$ we recover (2.7), and (2.8), respectively.

The Homogeneous sine-Gordon theories describe integrable perturbations of parafermionic theories defined by an action of the form (1.1) [12, 14], and we can investigate the continuation $\mu \rightarrow-\mu$ using the methods of section 3. The HSG TBA equations (4.6) with the energy terms (4.7) provide solutions to the Y-system (2.9), for $H=A_{k-1}$, with the asymptotic behaviour

$$
\begin{array}{r}
Y_{a}^{i}(r, \theta) \stackrel{\theta \rightarrow-\infty}{\longrightarrow} \exp \left(\frac{1}{2} m_{i}^{+} \mu_{a} r \mathrm{e}^{-\theta}\right) \\
\stackrel{\theta \rightarrow+\infty}{\longrightarrow} \exp \left(\frac{1}{2} m_{i}^{-} \mu_{a} r \mathrm{e}^{+\theta}\right) .
\end{array}
$$

Consequently, they are completely characterised by the value of the parameters $m_{i}^{+}$ and $m_{i}^{-}$, for $i=1 \ldots r_{G}$. Then, the results of section 3 imply that the continuation $\mu \rightarrow-\mu$ makes the $\mathrm{Y}$-functions change according to

$$
Y_{a}^{i}(r, \theta) \rightarrow \tilde{Y}_{a}^{i}(r, \theta)=Y_{a}^{i}\left(\mathrm{e}^{i \frac{\pi P}{2}} r, \theta+i \frac{\pi P}{2}\right)=Y_{\bar{a}}^{\bar{\imath}}\left(\mathrm{e}^{i \frac{\pi P}{2}} r, \theta-i \frac{\pi P}{2}\right)
$$

In turn, taking (2.13) into account, the transformed Y-functions have the asymptotic behaviour

$$
\begin{array}{r}
\tilde{Y}_{a}^{i}(r, \theta) \stackrel{\theta \rightarrow-\infty}{\longrightarrow} \exp \left(\frac{1}{2} m_{i}^{+} \mu_{a} r \mathrm{e}^{-\theta}\right) \\
\stackrel{\theta \rightarrow+\infty}{\longrightarrow} \exp \left(\frac{1}{2} m_{\bar{\imath}}^{-} \mu_{a} r \mathrm{e}^{+\theta}\right) .
\end{array}
$$

Therefore, we conclude that the $\mu \rightarrow-\mu$ continuation of the HSG TBA system with parameters $\left\{m_{i}^{+}, m_{i}^{-}\right\}$is equivalent to the transformation

$$
m_{i}^{+} \rightarrow \widetilde{m}_{i}^{+}=m_{i}^{+}, \quad m_{i}^{-} \rightarrow \widetilde{m}_{i}^{-}=m_{\bar{\imath}}^{-}, \quad \forall i=1 \ldots r_{G} .
$$

Taking into account the relationship between the massless and massive magnonic systems and the HSG TBA equations described in the paragraph around eq. (4.9), 
this generalises the connection between the continuation $\mu \rightarrow-\mu$ and the transformation between massless and massive systems originally pointed out in [9], which was discussed in the previous section. To be specific, let us assume that the scale $m_{p p}$ is 'unshielded' and 'well separated' from the others, so that the HSG theory exhibits a crossover at $r \approx 2 / m_{p p}$. According to (4.10), the effective TBA system that describes this crossover is one of the massive magnonic TBA systems considered in section 2. Then, since

$$
m_{p p}=\sqrt{m_{p}^{+} m_{p}^{-}}=\sqrt{m_{p}^{+} \widetilde{m}_{\bar{p}}^{-}} \equiv \widetilde{m}_{p \bar{p}}
$$

the same theory with $\mu \rightarrow-\mu$ will exhibit a crossover at $r \approx 2 / \widetilde{m}_{p \bar{p}}$, which is now effectively described by a massless magnonic TBA system.

\section{The $\mu \rightarrow-\mu$ continuation and T-duality.}

We will now show that the equivalence between the continuation $\mu \rightarrow-\mu$ and the transformation (4.14) can be deduced in a completely different way using the original Lagrangian formulation of the HSG theories in terms of a gauged WZW action modified by a potential.

Let us denote by $g$ the Lie algebra of the group $G$. The theories corresponding to perturbations of the $G_{k} / U(1)^{r_{G}}$ coset CFT have actions 11, 12,

$$
S_{\mathrm{HSG}}\left[\gamma, A_{ \pm}\right]=k\left(S_{\mathrm{gWZW}}\left[\gamma, A_{ \pm}\right]-\int d^{2} x V(\gamma)\right)
$$

Here, $\gamma=\gamma(t, x)$ is a bosonic field that takes values in some faithful representation of the compact Lie group $G$, and $A_{ \pm}$are non-dynamical gauge fields taking values in the Cartan subalgebra of $g$ associated with $H \simeq U(1)^{r_{G}}$, a maximal torus of $G$. Then, $k S_{\text {gWZW }}$ is the gauged WZW action corresponding to the coset $G_{k} / H$. The potential is

$$
V(\gamma)=\frac{m_{0}^{2}}{4 \pi}\left\langle\Lambda_{+}, \gamma^{\dagger} \Lambda_{-} \gamma\right\rangle
$$

where $m_{0}^{2}$ is a bare overall mass scale, $\langle$,$\rangle is the Killing form of g$, and $\Lambda_{ \pm}=i \boldsymbol{\lambda}_{ \pm} \cdot \boldsymbol{h}$ are two arbitrary elements in the same Cartan subalgebra of $g$ where $A_{ \pm}$take values. They are specified by two $r_{G^{-}}$dimensional vectors $\boldsymbol{\lambda}_{+}$and $\boldsymbol{\lambda}_{-}$, and we will make this dependence explicit by writing

$$
V \equiv V\left[\boldsymbol{\lambda}_{+}, \boldsymbol{\lambda}_{-}\right]
$$

In [16], it was shown that there is a group of T-duality transformations that relate the HSG models corresponding to different values of $\boldsymbol{\lambda}_{ \pm}$. Namely, there is a duality transformation for each Weyl transformation $\sigma \in \mathcal{W}(G)$ that relates the models specified by the following potentials

$$
V\left[\boldsymbol{\lambda}_{+}, \boldsymbol{\lambda}_{-}\right] \stackrel{\text { T-duality }}{\longrightarrow} V\left[\boldsymbol{\lambda}_{+}, \sigma\left(\boldsymbol{\lambda}_{-}\right)\right]
$$


and provides a map between two different phases of the model. To spell this out, we have to be more precise about the possible values of $\boldsymbol{\lambda}_{+}$and $\boldsymbol{\lambda}_{-}$. The HSG theories are massive for any choice of $\boldsymbol{\lambda}_{+}$and $\boldsymbol{\lambda}_{-}$such that $\boldsymbol{\lambda}_{ \pm} \cdot \boldsymbol{\alpha} \neq 0$ for all the roots $\boldsymbol{\alpha}$ of $g$ [11]. This makes possible to choose the basis of simple roots of $g, \Delta=\left\{\boldsymbol{\alpha}_{1} \ldots \boldsymbol{\alpha}_{r_{G}}\right\}$, such that $\boldsymbol{\lambda}_{+} \cdot \boldsymbol{\alpha}_{i}>0$ for all $i=1 \ldots r_{G}$. In other words, without losing generality, we can restrict $\boldsymbol{\lambda}_{+}$to take values inside $C(\Delta)$, the fundamental Weyl chamber with respect to $\Delta$; i.e., $\boldsymbol{\lambda}_{+} \in C(\Lambda)$. Then, the different phases of the theory are characterised by the domain where $\boldsymbol{\lambda}_{-}$takes its values [16]. Since all the Weyl chambers are permuted by $\mathcal{W}(G)$, there is a phase for each Weyl transformation $\sigma \in \mathcal{W}(G)$ corresponding to $\boldsymbol{\lambda}_{-} \in \sigma^{-1}(C(\Delta))$, which justifies the interpretation of (5.4) as a map between two different phases.

In the semiclassical limit, the vectors $\boldsymbol{\lambda}_{+}$and $\boldsymbol{\lambda}_{-}$are related to the TBA parameters $\left\{m_{i}, \sigma_{i}\right\}$. Consider an arbitrary phase where $\boldsymbol{\lambda}_{-} \in \sigma^{-1}(C(\Delta))$. Then,

$$
\sigma\left(\boldsymbol{\lambda}_{-}\right)=\boldsymbol{\lambda}_{-}^{\bullet} \in C(\Delta)
$$

and the relationship is as follows [11, 14]

$$
\boldsymbol{\lambda}_{+}=\sum_{i=1}^{r_{G}} m_{i}^{+} \boldsymbol{\lambda}_{i}, \quad \boldsymbol{\lambda}_{-}^{\bullet}=\sum_{i=1}^{r_{G}} m_{i}^{-} \boldsymbol{\lambda}_{i},
$$

where $m_{i}^{ \pm}$are the semiclassical counterparts of the parameters defined in (4.8), and $\boldsymbol{\lambda}_{i}, i=1 \ldots r_{G}$, are the fundamental weights of $g$ that satisfy $\boldsymbol{\lambda}_{i} \cdot \boldsymbol{\alpha}_{j}=\delta_{i j}$. Notice that all the choices of $\boldsymbol{\lambda}_{-}$related by the T-duality transformations share the same $\boldsymbol{\lambda}_{-}^{\bullet}$. This is so because they have the same masses and resonance parameters, which can be seen as a semiclassical confirmation of the duality symmetry.

We can now easily describe the meaning of the continuation $\mu \rightarrow-\mu$ in this approach. In (5.1), $S_{\mathrm{HSG}}$ is a Lagrangian action defined on $1+1$ Minkowski space. This is in contrast with (1.1), which defines the model as a perturbed conformal field theory in two-dimensional Euclidean space with the role of the potential $k V(\gamma)$ being taken by the perturbing operator $\mu \Phi$. Therefore, in this framework, the continuation $\mu \rightarrow-\mu$ of (1.1) corresponds to $V\left[\boldsymbol{\lambda}_{+}, \boldsymbol{\lambda}_{-}\right] \rightarrow-V\left[\boldsymbol{\lambda}_{+}, \boldsymbol{\lambda}_{-}\right]$. Since

$$
-V\left[\boldsymbol{\lambda}_{+}, \boldsymbol{\lambda}_{-}\right]=V\left[\boldsymbol{\lambda}_{+},-\boldsymbol{\lambda}_{-}\right],
$$

this amounts to changing the phase of the model by means of $\boldsymbol{\lambda}_{-} \rightarrow-\boldsymbol{\lambda}_{-}$.

Let us consider a generic phase where $\sigma\left(\boldsymbol{\lambda}_{-}\right)=\boldsymbol{\lambda}_{-}^{\bullet} \in C(\Delta)$. In order to find out the effect of (5.7) on the TBA parameters, we have to look for a Weyl transformation that takes $\sigma\left(-\boldsymbol{\lambda}_{-}\right)$back into $C(\Delta)$; i.e., we have to look for $\sigma_{0} \in \mathcal{W}(G)$ such that

$$
\sigma_{0} \sigma\left(-\boldsymbol{\lambda}_{-}\right)=-\sigma_{0}\left(\boldsymbol{\lambda}_{-}^{\bullet}\right) \in C(\Delta)
$$

for any $\boldsymbol{\lambda}_{-}^{\bullet} \in C(\Delta)$. There is a unique Weyl transformation with this property: the so-called 'longest Weyl group element' . Its explicit form can be found, for instance, 
in [29, appendix E.13]. It can be written in a concise way via

$$
\sigma_{0}=-\omega_{0},
$$

where

$$
\omega_{0}=\left\{\begin{array}{l}
1, \quad \text { for } g=a_{1}, d_{2 n}, e_{7}, e_{8}, b_{n}, c_{n}, f_{4}, g_{2}, \\
\text { Dynkin diagram automorphism, otherwise. }
\end{array}\right.
$$

Notice that $\omega_{0}$ acts on the Dynkin diagram $G$. In particular for simply-laced $G$,

$$
\omega_{0}(i)=\bar{\imath} \quad \forall i=1 \ldots r_{G},
$$

where $\bar{\imath}$ is the conjugation defined in fig. 1.

Therefore, we conclude that the continuation $\mu \rightarrow-\mu$ of the HSG action is equivalent to the transformation $\boldsymbol{\lambda}_{-}^{\bullet} \rightarrow \omega_{0}\left(\boldsymbol{\lambda}_{-}^{\bullet}\right)$. This amounts to

$$
m_{i}^{+} \rightarrow m_{i}^{+}, \quad m_{i}^{-} \rightarrow m_{\bar{\imath}}^{-}, \quad \forall i=1 \ldots r_{G},
$$

which coincides with the equivalence (4.14), deduced above using the Y-system continuation argument of [9, 10]. The agreement between the two methods used to deduce (4.14) and (5.12) is one of the main results of this letter.

\section{Conclusions}

The main result of this letter is the derivation of a new Lagrangian interpretation of the relationship between the transformation of massive into massless magnonic TBA systems, and the change of sign of the coupling constant in the corresponding perturbed conformal field theory actions. This relationship was originally noticed by Al. Zamolodchikov in the study of the perturbation of the unitary minimal models by their least relevant primary field [7], and it was found to be true in many other

cases. Its extent was clarified in [9, 10], where it was related to the properties of the associated Y-system by means of the study of the analytic continuation of the TBA equations.

The novel interpretation arises in the context of the Homogeneous sine-Gordon (HSG) theories [11, 12, 13], whose TBA equations are purely massive generalisations of the magnonic TBA systems corresponding to products of Dynkin diagrams of the form $G \times A_{k}$. They depend on $2 \operatorname{rank}(G)-1$ independent adjustable parameters, and the usual magnonic massive and massless systems are recovered as the effective TBA systems that describe the crossovers of these theories for particular limiting values of those parameters [14]. The HSG theories admit a Lagrangian formulation in terms of a gauged Wess-Zumino-Witten action with a potential term that takes the role of the perturbing operator in their interpretation as perturbed conformal field theories. 
In this framework, the relationship arises as a consequence of the target-space ( $\mathrm{T}$ space) duality symmetries of the Lagrangian action [16. To be specific, changing the sign of the coupling constant corresponds to an overall change of the sign of the potential, which is T-dual to a transformation among the adjustable parameters. This transformation changes the pattern of crossovers exhibited by the theory in such a way that all the crossovers effectively described by massive magnonic TBA systems turn out to be described by massless ones and vice versa, thus giving support to the claim that the observed relationship can indeed be understood as a consequence of T-duality.

Moreover, we have shown that the transformation among the adjustable parameters, summarised by (4.14) and (5.12), can also be obtained through the generalisation of the arguments of [9, 10] to study the analytic continuation of the HSG TBA system. This is rather remarkable because the two methods used to deduce it are expected to be valid in different regimes. Namely, eq. (4.14) was derived from the analytical continuation of the HSG TBA system, making use of the properties of the corresponding Y-systems. This is expected to hold for values of the dimensionless overall scale $r$ smaller than some upper value $r_{0}$, which means that we should be close enough to the UV limit. In contrast, the Lagrangian arguments leading to $(5.12)$ should be valid in the semiclassical, large $k$, limit. The agreement of the resulting transformations provides a second interpretation of our results, as a non-perturbative check, enabled by integrability, of the semiclassical Lagrangian arguments used in [16, 30] to study T-duality in a particular family of massive theories (for a different class of models, integrability was previously used for similar purposes in [31]). Conversely, we expect that T-duality symmetries will provide relevant information to understand better the nature of the flows in the HSG models, and the relationship between their TBA and Lagrangian parameters.

\section{Acknowledgments}

We would like to thank Clare Dunning, Francesco Ravanini and Roberto Tateo for helpful discussions, and collaborations on many closely-related topics. JLM thanks Bologna (INFN and University) for hospitality. This work was partly supported by the EC network "EUCLID", contract number HPRN-CT-2002-00325, and partly by a NATO grant PST.CLG.980424. JLM also thanks MEC (Spain) and FEDER (FPA2005-00188 and FPA2005-01963), and Incentivos from Xunta de Galicia for financial support. 


\section{References}

[1] Al.B. Zamolodchikov, 'Thermodynamic Bethe ansatz in relativistic models: scaling 3-state Potts and Lee-Yang models', Nucl. Phys. B 342 (1990) 695.

[2] A.B. Zamolodchikov, 'Integrable field theory from conformal field theory', $A d v$. Stud. Pure Math. 19 (1989) 641.

[3] F. Ravanini, R. Tateo, and A. Valleriani, 'Dynkin TBAs', Int. J. Mod. Phys. A 8 (1993) 1707, hep-th/9207040;

E. Quattrini, F. Ravanini, and R. Tateo, 'Integrable $\mathrm{QFT}_{2}$ encoded on products of Dynkin diagrams', hep-th/9311116.

[4] M.J. Martins, 'The Thermodynamic Bethe Ansatz For Deformed $W A_{N-1}$ Conformal Field Theories', Phys. Lett. B 277 (1992) 301, hep-th/9201032.

[5] F. Ravanini, 'Thermodynamic Bethe ansatz for $\mathcal{G}_{k} \otimes \mathcal{G}_{l} / \mathcal{G}_{k+l}$ coset models perturbed by their $\phi_{1,1, A d j}$ operator, Phys. Lett. B 282 (1992) 73, hep-th/9202020.

[6] Al.B. Zamolodchikov, 'Thermodynamic Bethe ansatz for RSOS scattering theories', Nucl. Phys. B 358 (1991) 497.

[7] Al.B. Zamolodchikov, 'From tricritical Ising to critical Ising by Thermodynamic Bethe ansatz', Nucl. Phys. B 358 (1991) 524.

[8] V.A. Fateev and Al.B. Zamolodchikov, 'Integrable perturbations of $\mathbb{Z}_{N}$ parafermion models and the $O(3)$ sigma models', Phys. Lett. B 271 (1991) 91.

[9] P. Dorey, C. Dunning and R. Tateo, 'New families of flows between two-dimensional conformal field theories', Nucl. Phys. B 578 (2000) 699, hep-th/0001185.

[10] P. Dorey, C. Dunning and R. Tateo, unpublished.

[11] C.R. Fernández-Pousa, M.V. Gallas, T.J. Hollowood and J.L. Miramontes, 'The symmetric space and homogeneous sine-Gordon theories', Nucl. Phys. B 484 (1997) 609, hep-th/9606032.

[12] C.R. Fernández-Pousa, M.V. Gallas, T.J. Hollowood and J.L. Miramontes, 'Solitonic integrable perturbations of parafermionic theories', Nucl. Phys. B 499 (1997) 673, hep-th/9701109.

[13] J.L. Miramontes and C.R. Fernández-Pousa, 'Integrable quantum field theories with unstable particles', Phys. Lett. B 472 (2000) 392, hep-th/9910218.

[14] P. Dorey and J.L. Miramontes, 'Mass scales and crossover phenomena in the Homogeneous Sine-Gordon Models', Nucl. Phys. B 697 (2004) 405,

hep-th/0405275. 
[15] O.A. Castro-Alvaredo, A. Fring, C. Korff and J.L. Miramontes, 'Thermodynamic Bethe ansatz of the homogeneous sine-Gordon models', Nucl. Phys. B 575 (2000) 535 , hep-th/9912196.

[16] J.L. Miramontes, 'T-duality in massive integrable field theories: the Homogeneous and complex sine-Gordon models', Nucl. Phys. B 702 (2004) 419, hep-th/0408119.

[17] H.W. Braden, E. Corrigan, P.E. Dorey and R. Sasaki, 'Affine Toda field theory and exact $S$-matrices', Nucl. Phys. B 338 (1990) 689;

P. Dorey and F. Ravanini, 'Staircase models from affine Toda field theory', Int. J. Mod. Phys. A 8 (1993) 873, hep-th/9206052; 'Generalizing the staircase models', Nucl. Phys. B 406 (1993) 708, hep-th/9211115.

[18] P. Dorey, R. Tateo and K.E. Thompson, 'Massive and massless phases in self-dual $Z_{N}$ spin models: some exact results from the thermodynamic Bethe ansatz', Nucl. Phys. B 470 (1996) 317, hep-th/9601123.

[19] Al.B. Zamolodchikov, 'On the thermodynamic Bethe ansatz equations for reflectionless ADE scattering theories', Phys. Lett. B 253 (1991) 391.

[20] A. Klümper and P.A. Pearce, 'Analytical calculations of scaling dimensions: tricritical hard square and critical hard hexagons', J. Statist. Phys. 64 (1991) 13; 'Conformal weights of RSOS lattice models and their fusion hierarchies', J. Phys. A 183 (1992) 304;

G. Jüttner, A. Klümper and J. Suzuki, 'From fusion hierarchy to excited state TBA', Nucl. Phys. B 512 (1998) 581;

A. Kuniba, K. Sakai and J. Suzuki, 'Continued fraction TBA and functional relations in XXZ model at root of unity', Nucl. Phys. B 525 (1998) 597.

[21] M.J. Martins, 'Complex excitations in the thermodynamic Bethe ansatz approach', Phys. Rev. Lett. 67 (1991) 419;

V.V. Bazhanov, S.L. Lukyanov and A.B. Zamolodchikov, 'Quantum field theories in finite volume: Excited state energies" Nucl. Phys. B 489 (1997) 487,

hep-th/9607099;

J. Balog and A. Hegedus, 'TBA equations for excited states in the sine-Gordon model', J. Phys. A 37 (2004) 1903, hep-th/0304260.

[22] P. Dorey and R. Tateo, 'Excited states by analytic continuation of TBA equations', Nucl. Phys. B 482 (1996) 639, hep-th/9607167.

[23] P. Dorey and R. Tateo, 'Excited states in some simple perturbed conformal field theories', Nucl. Phys. B 515 (1998) 575, hep-th/9706140.

[24] F. Gliozzi and R. Tateo, 'ADE functional dilogarithm identities and integrable models', Phys. Lett. B 348 (1995) 84, hep-th/9411203.

[25] F. Gliozzi and R. Tateo, 'Thermodynamic Bethe ansatz and threefold triangulations', Int. J. Mod. Phys. A 11 (1996) 4051, hep-th/9505102; 
E. Frenkel and A. Szenes, 'Thermodynamic Bethe ansatz and dilogarithm identities. 1', Math. Res. Lett. 2 (1995) 677, hep-th/9506215.

[26] R. Caracciolo, F. Gliozzi and R. Tateo, 'A topological invariant of RG flows in 2D integrable quantum field theories', Int. J. Mod. Phys. B 13 (1999) 2927, hep-th/9902094.

[27] S. Fomin and A. Zelevinsky, 'Y-systems and generalized associahedra', Ann. Math. 158 (2003) 977, hep-th/0111053.

[28] A.Y. Volkov, 'On Zamolodchikov's periodicity conjecture for Y-systems', hep-th/0606094;

A. Szenes, 'Periodicity of Y-systems and flat connections', math.RT/0606377.

[29] J.F. Cornwell, 'Group theory in physics (Vol. II)', Academic Press, 1984.

[30] J.F. Gomes, E.P. Gueuvoghlanian, G.M. Sotkov and A.H. Zimerman, 'T-duality of axial and vector dyonic integrable models', Ann. Phys. (NY) 289 (2001) 232,

hep-th/0007116;

J.F. Gomes, G.M. Sotkov and A.H. Zimerman, 'Axial vector duality in affine NA Toda models', in 'Workshop on Integrable Theories, Solitons and Duality', PrHEP-unesp2002/045, hep-th/0212046; 'T-duality in 2D integrable models', J. Phys. A 37 (2004) 4629, hep-th/0402091

[31] J. Balog, P. Forgacs and L. Palla, 'A two-dimensional integrable axionic sigma model and T-duality', Phys. Lett. B 484 (2000) 367, hep-th/0004180;

P. Forgacs, 'A 2-D integrable axion model and target space duality', in 'Budapest 2000, Non-perturbative QFT methods and their applications', hep-th/0111124. 